\title{
Conocimiento y actitudes sobre el consumo de bebidas alcohólicas de los estudiantes de las universidades de la Región Lima - 2014
}

\author{
Knowledge and attitudes about drinking students from universities in the Region - 2014 \\ Elsa Carmen Oscuvilca Tapia ${ }^{1, a}$, Walter Jesús Sosa Hijar ${ }^{1, b}$, Olga Cáceres Estrada, ${ }^{1, c}$, \\ William Rogelio Peña Ayudante ${ }^{1, d}$, Jacinto Jesús Palacios Solano ${ }^{1, e}$
}

\begin{abstract}
RESUMEN
Objetivo: Determinar la relación entre el conocimiento y las actitudes sobre el consumo de bebidas alcohólicas de los estudiantes de las universidades de la Región Lima - 2014. Materiales y Métodos: Estudio descriptivo correlacional, transversal, retrospectivo realizado en una muestra de 234 estudiantes de las universidades de la Región Lima; cuenta con el soporte de las teorías de Ajzen y Fishbein y Bandura; los instrumentos para la recolecta de datos válidos y confiables mediante el juicio de expertos y prueba piloto respectivamente. Para el análisis de los datos se empleó la prueba Chi cuadrado con el programa SPSS versión 17.0. Resultados: EI $45,7 \%$ de estudiantes universitarios tienen el conocimiento alto; el $38,9 \%$ conocimiento medio y el $15,4 \%$ el conocimiento bajo. La actitud de los universitarios ante el consumo de bebidas alcohólicas es indiferente en el $50 \%$ negativa en el $27,4 \%$ y positiva en el $22,6 \%$. Conclusión: La relación significativa entre el conocimiento alto y la actitud indiferente de los estudiantes universitarios ante el consumo de bebidas alcohólicas, genera una línea de investigación en el área de salud mental, en procura de erradicar la cultura del consumo de alcohol por influencia de redes sociales, por encontrarse en un periodo idóneo para la adopción de hábitos y actitudes, mediante la observación e imitación.
\end{abstract}

Palabras clave: Promoción de salud, universidad saludable, patrones de consumo bebida alcohólica.

\begin{abstract}
Objective: To determine the relationship between knowledge and attitudes about the consumption of alcoholic beverages of the students of the universities of the Region Lima - 2014. Materials and Methods: Descriptive, cross-sectional, retrospective descriptive study carried out on a sample of 234 students from the Universities of the Lima Region; Has the support of the theories of Ajzen and Fishbein and Bandura; The instruments for the collection of valid and reliable data through expert judgment and pilot testing respectively. For the analysis of the data the Chi square test was used with the program SPSS version 17.0. Results: $45.7 \%$ of university students have high knowledge; $38.9 \%$ average knowledge and $15.4 \%$ low knowledge. The attitude of university students to the consumption of alcoholic beverages is indifferent in $50 \%$ negative in $27.4 \%$ and positive in $22.6 \%$. Conclusion: The significant relationship between the high knowledge and the indifferent attitude of university students to the consumption of alcoholic beverages, generates a line of research in the area of mental health, in an attempt to eradicate the culture of alcohol consumption by influence of networks Social, for being in an ideal period for the adoption of habits and attitudes, through observation and imitation.
\end{abstract}

Key words: Health promotion, healthy university, consumption patterns alcoholic consumption.

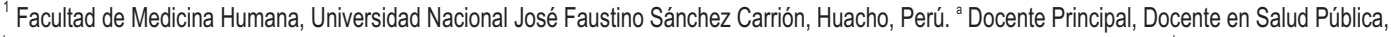

${ }^{\circ}$ Docente Asociado. Licenciado en Bromatología y Nutrición, ${ }^{\circ}$ Docente Principal Mstra. Investigación y Docencia Universitaria, ${ }^{\circ}$ Docente Asociado. Maestro en Salud Reproductiva,

${ }^{e}$ Docente Principal. Magíster en Fisiología. 


\section{INTRODUCCIÓN}

Se calcula que el uso nocivo del alcohol causa cada año 2,5 millones de muertes, y una proporción considerable de ellas corresponde a personas jóvenes. El consumo de bebidas alcohólicas ocupa el tercer lugar entre los principales factores de rriesgo de inadecuada salud en el mundo, Una gran variedad de problemas relacionados con el alcohol pueden tener repercusiones devastadoras en las personas y sus familias; además, pueden afectar gravemente a la vida comunitaria. El uso nocivo del alcohol es uno de los cuatro factores de riesgo de enfermedades no transmisibles importantes que son susceptibles de modificación y prevención. También están surgiendo indicios de que dicho uso contribuye a aumentar la carga de morbilidad relacionada con enfermedades transmisibles como, por ejemplo, la tuberculosis y la infección por el VIH/SIDA. El uso nocivo del alcohol ocupa el tercer lugar entre los principales factores de riesgo de muerte prematura y discapacidad en el mundo. Se calcula que en 2004 murieron por causas relacionadas con el alcohol unos 2,5 millones de personas en todo el mundo, entre ellas 320000 jóvenes entre los 15 y los 29 años de edad. (OMS, 2010)

El consumo de alcohol se ha expandido en forma generalizado en la mayoría de los países del continente americano, se ha demostrado que el promedio de consumo de alcohol per cápita en América es de 8,9 litros, supera el consumo mundial que alcanza los 5,8 litros. Bolivia ocupa el cuarto lugar en consumo de alcohol en América Latina, después de México, República Dominicana y Panamá (Monteiro, 2007)

En el Perú, la prevalencia de vida del consumo de drogas sociales (alcohol y tabaco) es mayor en el nivel socioeconomico alto, los fines de semana frecuentan ciertos lugares de oferta de las bebidas alcohólicas, como bares, discotecas, fiestas electrónicas, influenciados por el grupo de amigos. (Rojas, 2002).

Debido a que no hay restricciones en la venta de licor, hay un consumo masivo porque el alcohol es droga legal. En el año 2010, más de 1,5 millones de peruanos presentaron problemas con el alcohol, adicción que representa la segunda causa de enfermedades y muertes en el país (MINSA, 2010).

Por lo observado se formula el problema ¿Qué relación existe entre el conocimiento y las actitudes sobre el consumo de bebidas alcohólicas de los estudiantes de las universidades de la Región Lima 2014. La presente investigación se justifica por la relevancia social, debido a que el conocimiento y actitud ante el consumo de bebidas alcohólicas de los estudiantes universitarios obliga el abordaje transdisciplinario ante la necesidad de desarrollar un marco establecido por la Organización Mundial de la Salud, la investigación dirigida al grupo vulnerable logrará predecir la calidad de vida durante años subsecuentes, con el propósito de disminuir las tasas de morbi mortalidad, no olvidemos que, en pocos años se constituirán en población económicamente activa.

El soporte teórico de la Acción Razonada de Ajzen y Fishbein (1980) y Aprendizaje social de Bandura (1986) contribuyen a buscar nuevas lineas de investigación en el área de salud mental de los universitarios, y el logro del objetivo general, determinar la relación entre el conocimiento y las actitudes sobre el consumo de bebidas alcohólicas de los estudiantes de las universidades de la Región Lima- 2014.

\section{MATERIALES Y MÉTODOS}

Tipo de investigación. Según el problema y los objetivos, la investigación fue de enfoque cuantitativo cualitativo, tipo descriptivo correlacional. De acuerdo al tiempo de ocurrencia de los hechos y registro de la información fue retrospectiva y transversal.

Área de estudio: Se desarrolló en las universidades públicas de la Región Lima.

Población: 12,525 universitarios (12, 200 de la Universidad Nacional José Faustino Sánchez Carrión y 325 de la Universidad Nacional de Barranca).

Tamaño de Muestra: 234 estudiantes universitarios determinados con la fórmula para población finita y nivel de confianza deseado.

\begin{tabular}{|cc|}
\hline $\mathrm{N}$ & 12525 \\
\hline $\mathrm{Z}$ & 1.555 \\
\hline $\mathrm{p}$ & 0.5 \\
\hline $\mathrm{q}$ & 0.5 \\
\hline $\mathrm{E}$ & 0.05 \\
\hline $1-\infty$ & $88 \%$ \\
\hline Error Muestral $(\mathrm{E})$ & $5 \%$ \\
\hline
\end{tabular}

tamaño muestra ajustado: $\mathrm{n}=\mathrm{no} / 1+(\mathrm{no} / \mathrm{N})$

\begin{tabular}{|l|c|c|}
\hline Tamaño muestra & no $=$ & 237 \\
Muestra ajustada & no & 234 \\
\hline
\end{tabular}

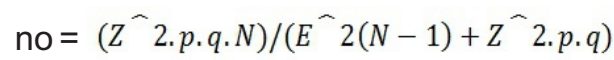

Tipo de muestreo: Aleatorio simple.

\section{Criterios de inclusión}

* Universitarios matriculados en el semestre académico 2014 - II.

* Universitarios que accedan a participar en la investigación. 


\section{Criterios de exclusión}

* Universitarios con asistencia libre o no regular,

* Universitarios que accedan a participar en la investigación.

Técnicas e instrumentos de recolección de datos

La encuesta y el cuestionario de tipo semi estructurado, que corresponde los ítems del conocimiento sobre consumo de bebidas alcohólicas, el puntaje asignado es: respuesta correcta $=1 \mathrm{y}$ la respuesta incorrecta $=0$. Para recolectar la información de las Actitudes, el instrumento, los ítems no requieren de respuestas correctas ni incorrectas, debido a que los enunciados expresan las creencias, los sentimientos y las tendencias del universitario. La tendencia de las actitudes en este caso fue descrita por medio de la propiedad del grado del sentimiento (por la intensidad) distribuidos en la escala de Lickert representado por:

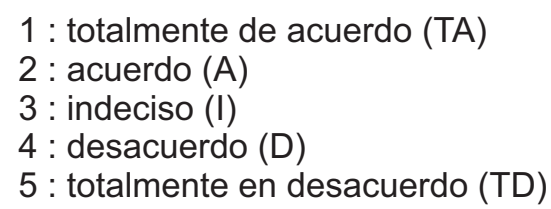

Confiabilidad del instrumento: Se aplicó la prueba piloto a los universitarios de la universidad Mayor de San Marcos por reunir características similares a la población de estudio.

El instrumento para medir el conocimiento tiene:

Coeficiente de confiabilidad de las mitades según Spearman - Brown

0,701

Coeficiente de confiabilidad de las mitades según Rulon - Guttman

0,670

Coeficiente de confiabilidad según la fórmula 20 de Kuder-Richardson (KR20)

Coeficiente de confiabilidad según la fórmula 21 de Kuder - Richardson (KR21)

Coeficiente de confiabilidad según la fórmula KR21 modificada por Horst

0,508

Para medir la actitud tiene los:

Coeficiente de confiabilidad de las mitades según Spearman - Brown

0,780

Coeficiente de confiabilidad de las mitades según Rulon - Guttman

0,779

Coeficiente de confiabilidad Alfa de Cronbach

0,826

Validez del instrumento: Se determinó mediante el juicio de expertos en el área de Enfermeria, Psicologia, Sociologia y Medicina Humana, con el coeficiente $\mathrm{R}$ de Finnque determinó el acuerdo fortísimo de 0.933 .

Los procedimientos para la recolección de información, fueron autorizados por las autoridades y por el sujeto de investigación, a través del consentimiento informado.

Técnicas, análisis y procedimientos de datos

La información codificada mediante la construcción de un libro de códigos, fue ingresada a Excel y exportada al sofware estadistico SPSS versión 17.0 (StacticalPakageforthr Social Sciencies) para Windows XP; La estadística descriptiva identifico el conocimiento y actitudes. La relación de las variables se determinó mediante la prueba chi cuadrado. La información se presentó en cuadros de una o doble entrada con sus respectivos gráficos.

\section{RESULTADOS}

Se incluyeron en el estudio 234 universitarios, de los cuales el $56,8 \%$ fueron mujeres y el $43,2 \%$ varones. La media de edad fue de 23 años, concentrados mayoritariamente en el III y IV ciclo. El $45,7 \%$ pertenecen a las carreras de Ingeniería, el 20,9\% a Ciencias Sociales, el 18,8\% a Ciencias de la Salud y el $14,5 \%$ a Ciencias Económicas, Financieras y Educación.

El conocimiento sobre el consumo de bebidas alcohólicas de los estudiantes de las universidades de la Región Lima, 107 (45,7 \%) tiene el conocimiento alto; $91(38,9 \%)$ conocimiento medio y $36(15,4 \%)$ conocimiento bajo.

Las actitudes ante el consumo de bebidas alcohólicas de los estudiantes de las universidades de la Región Lima es indiferente para $117(50 \%)$, negativa para 64 $(27,4 \%)$ y positiva para $53(22,6 \%)$

La relación entre el conocimiento y las actitudes sobre el consumo de bebidas alcohólicas de los estudiantes de las universidades de la Región Lima resultó significativa debido a que la prueba no paramétrica Chi cuadrado $=15,390, \mathrm{gl}=4, \mathrm{p}=0.004$. (Tabla 1)

Tabla 1. Relación entre el conocimiento y las actitudes sobre el consumo de bebidas alcohólicas de los estudiantes de las universidades de la Región Lima - 2014.

\begin{tabular}{cccccc}
\hline \multirow{2}{*}{ Conocimiento } & \multicolumn{3}{c}{ Actitudes } & \multirow{2}{*}{ Total } \\
\cline { 2 - 5 } & & Negativa & Indiferente & Positiva & \\
\hline \multirow{2}{*}{ Bajo } & $\mathrm{n}$ & 7 & 12 & 17 & 36 \\
& $\%$ & $3.0 \%$ & $5.1 \%$ & $7.3 \%$ & $15.4 \%$ \\
\multirow{2}{*}{ Medio } & $\mathrm{n}$ & 25 & 47 & 19 & 91 \\
& $\%$ & $10.7 \%$ & $20.1 \%$ & $8.1 \%$ & $38.9 \%$ \\
\multirow{2}{*}{ Alto } & $\mathrm{n}$ & 32 & 58 & 17 & 107 \\
& $\%$ & $13.7 \%$ & $24.8 \%$ & $7.3 \%$ & $45.7 \%$ \\
\multirow{2}{*}{ Total } & $\mathrm{n}$ & 64 & 117 & 53 & 234 \\
& $\%$ & $27.4 \%$ & $50.0 \%$ & $22.6 \%$ & $100.0 \%$ \\
\hline
\end{tabular}

Fuente: Estudiantes de las Universidades de la Región Lima, 2014.

Análisis e interpretación de los datos

Las frecuencias observadas se sometiron a la 
siguiente prueba de hipótesis:

Ho: No existe relación entre el conocimiento y la actitud sobre el consumo de bebidas alcohólicas en los estudiantes de la Región Lima.

H1: Existe relación entre el conocimiento y la actitud sobre el consumo de bebidas alcohólicas en los estudiantes de la Región Lima.

Nivel de significancia: Error tipo I, $\alpha=0.05$ (5\%). Esto es, el error que se cometeria al rechazar la Ho, siendo esta verdadera.

Prueba Estadística: Prueba no paramétrica Chi cuadrado.

Decisión Estadistica: El chi cuadrado observado es $\mathrm{Ji}^{2}=15.390$ con $\mathrm{GL}=4$, al cual le está asociado un valor de probabilidad, $\mathrm{P}=0.004$. Puesto que este valor $P$ es menor o igual que $\alpha$, se concluye en rechazar la Ho. Es decir existe relación entre el conocimiento y la actitud sobre el consumo de bebidas alcohólicas en los estudiantes de la Región Lima.

COEFICIENTES DE CORRELACION SOMERS, KENDALL'S TAU-B, KENDALL'TAU-C Y GAMMA

Medidas de asociación direccionales (d de Somers)

\begin{tabular}{lllllll}
\hline & & Valor & $\begin{array}{c}\text { Asymp. Std. } \\
\text { Error }^{\mathrm{a}}\end{array}$ & Approx. $\mathrm{T}^{\mathrm{b}}$ & Approx. Sig. \\
\hline Oridnal vs & Somers' & Simétrico & -0.151 & 0.061 & -2.454 & 0.014 \\
Ordinal & $\mathrm{d}$ & Conocimiento & -0.150 & 0.061 & -2.454 & 0.014 \\
& & Actitudes & -0.152 & 0.061 & -2.454 & 0.014 \\
\hline
\end{tabular}

a. Asumiendo la hipótesis alternativa.

b. Empleando el error típico asintótico basado en la hipótesis nula.

Medidas simétricas

\begin{tabular}{llcccc}
\hline & Valor & $\begin{array}{c}\text { Asymp. Std. } \\
\text { Error }^{\text {a }}\end{array}$ & Approx. $\mathrm{T}^{\mathrm{b}}$ & Approx. Sig. \\
\hline Oridnal vs & Kendall's tau-b & -0.151 & 0.061 & -2.454 & 0.014 \\
Ordinal & Kendall's tau-c & -0.140 & 0.057 & -2.454 & 0.014 \\
N de casos validos & Gamma & -0.238 & 0.094 & -2.454 & 0.014 \\
\hline
\end{tabular}

a. Asumiendo la hipótesis alternativa.

b. Empleando el error típico asintótico basado en la hipótesis nula.

En conclusión podemos afirmar: Dado que el valor de las medidas en negativo y significativo (relación negativa) se puede afirmar que a medida que el conocimiento sobre bebidas alcohólicas tiende de medio a alto las actitudes tienden de indiferente a negativa.

\section{DISCUSIÓN}

Los resultados permiten evidencias en los universitarios el conocimiento alto y la actitud indiferente ante el consumo de bebidas alcohólicas, debido a que no tienen el hábito de consumir bebidas alcohólicas, con frecuencia o en exceso, aun cuando señalan una percepcion del contexto como altamente consumidor, ya sea entre sus pares como en los adultos. Respecto a la peligrosidad del abuso frecuente de bebidas alcohólicas para la salud, consideran que es dañino para la salud. Los motivos para el consumo, un $49 \%$ manifiestan que la influencia del grupo o redes sociales tiene un peso mayor, así mismo por imitación y motivos personales, escenario similar sucede en los universitarios del Nordeste Argentino Instituto de Criminalistica y Criminologia en Corrientes de Argentina. (Porciel 2011)

Las marcadas diferencias de género en cuanto al consumo, los varones adjudican el alivio de la tensión psicológica a los problemas relacionados con frustraciones en los económico y con la pareja, las mujeres refieren que el consumo de bebidas alcohólicas incrementa la sexualidad en sus parejas y la agresividad, situación que se evidencia en los universitarios de la ciudad de Mexico. (Camacho, 2013) y en los de Pamplona de Colombia. (Mantilla Toloza, 2011) consideran a la bebida alcohólica, como droga legal y es la mas consumida acompañada con tabaco, en las fiesta y reuniones con sus pares.

No coincide de Beleño (2011), Sánchez (2002) y Urbina (2011) en la definición de bebidas alcohólicas, pues ignoran del compuesto del etanol (alcohol etílico) y desconocen los efectos nocivos e irreversibles en el organismo humano, desconocen que fisiológicamente el cerebro humano continua desarrollandose hasta aproximadamente los primeros años de la veintena y que un cerebro en desarrollo expuesto al consumo de alcohol puede sufrir efectos perdurables sobre las capacidades intelectuales y que pueden incrementarse las probabilidades de la adicción al alcohol y si se encuentran embarazadas el pronóstico es reservado.

Las teorías que soportan esta investigación demuestran que el estudiante universitario habitualmente vive el periodo transicional de la infancia a la edad adulta, en ella se establece la diferenciación especifica de todos los aspectos del desarrollo, físico, mental, moral y social, es un periodo idóneo para la adopción de hábitos y actitudes, especialmente en el uso de sustancias adictivas que posteriormente pueden permanecer en la adultez, si no son bien manejadas, y llegan a afectar la salud individual y la sociedad, porque establecen relaciones comunicativas comprensivas con los grupos y con los grupos y con los diferentes colectivos humanos, esta experiencia se asocia al conjunto de creencias, sentimientos y tendencias para dar lugar a un determinado actitud o comportamiento; mediante la observación e imitación. (Ajzen y Fishbein, 1980), (Bandura, 1986). Las redes sociales que empieza a tejer el universitario son construidas a partir de las asociaciones estimulo - respuesta y ellas son las que en definitiva determinan el aprendizaje cuyo contenido es cognitivo. (Garcia 2005), (Gonzalez 2004). El desarrollo cognitivo, evoluciona desde la capacidad de entender y construir temas y conceptos abastractos. Entre los 17 y 18 años de capacidad de inteligencia, llega a su totalidad, entiende como un 
adulto, adquiere varias capacidades importantes: toma sus propias decisiones y razona acerca del mismo, soluciona no un solo problema de la vida cotidiana, puede solucionar varios a la vez, comienza a tener nuevas facultades de pensamiento y se vuelve introspectivo, analítico y autocrítico. En las actitudes del universitario intervienen tres elementos fundamentales: La información que se recibe; el grupo de amigos con los que se identifica, quienes aportan a la formación de actitudes; y por ultimo, las necesidades personales del adolescente.

Los resultados indican que existe la necesidad de reformular políticas sociales (OMS 2007) entorno a los objetivos dirigidos a reducir tanto el consumo de alcohol de los jóvenes como los daños derivados del mismo, para lo cual se proponen medidas eficaces de política de alcohol en cuanto amplas áreas: proveer protección, promover la educación , reducir el daño y apoyar ambientes saludables. OMS (2011), debido a que el consumo juvenil del alcohol presenta hoy características propias que han generado una "cultura del consumo de alcohol" diferenciada del consumo tradicional. Esta situación tiene una triple dimensión: desde el adolescente, desde la sustancia, en este caso el alcohol, y desde el contexto actual: los valores de nuestra sociedad, el sistema productivo, la publicidad, el papel de la familia, de la escuela, de los grupos de iguales, bajo rendimiento escolar, agresiones, violencias, alteraciones del orden público y conductas de alto riesgo, como conducir tras haber bebido, asi como actividades sexuales de riesgo que conllevan embarazos no deseados y enfermedades de transmisión sexual, etc.

Se concluye que la relación es significativa entre el conocimiento alto y la actitud indiferente de los estudiantes universitarios ante el consumo de bebidas alcoholicas, el mismo que genera una linea de investigacion en el area de salud mental, en procura de erradicar la cultura del consumo de alcohol por influencia de redes sociales.

Conflicto de interés: Los autores declaran no tener conflictos de interés de ninguna naturaleza.

\section{AGRADECIMIENTO}

Los investigadores agradecen por su colaboración al Dr. Armando Vásquez Aristizabal por su colaboración en aplicaciones del instrumento de recolecta de datos. 


\section{REFERENCIAS BIBLIOGRÁFICAS}

Ajzen, I. y Fishbein. M. (1980). La comprensión de las actitudes y predecir sociales comportamiento. EnglewoodCliffs, NJ: prentice-Hall.

Bennasar M. (2012). Estilos de vida y salud en estudiantes universitarios: la universidad como entorno promotor de la salud. Universitat de les Illes Balears. España. Recuperado el 21 de Julio de 2014.

Beleño M. (2011). Impactos de la salud del individuo en paises de America Latina. Años 2006-2010.

Bandura A. (1986). Fundamentos sociales de pensamiento y acción. A sociales la teoria cognitiva. EnglewoodCliffs, NJ: Prentice Salas (trad. elenco. Barcelona, Martinez Roca, 1987)

Camacho I. (2005). Consumo de alcohol en universitarios: relacion funcional con los factores socio demograficos, las expectativas y la ansiedad social.

Garcia JE. (2005). Atención al adolescente. En: Álvarez Sintes, R. Temas de Medicina General Integr. La Habana: Edit Ciencias Médicas; p. 15962.

González R. (2004). Alcohol y otras drogas, Santiago de Cuba: Edith Oriente.

Monteir, Maristela G. (2007). Alcohol y salud publica en las Americas: un caso para acción. Washington, D.C: OPS.

Mantilla - Tolorza Sonia; Gomez - Conesa Antonia; Hidalgo - Montesinos Maria (2011). Antividad fisica, tabaquismo y consumo de alcohol, en un grupo de universitarios. Eniversidad de Pamplona, Colombia. 2011.

Ministerio de Salud del Perú (2010).

Organización Mundial de la Salud (2001) Declaraciones sobre Jovenes y Alcohol. Conferencia Ministerial sobre Jovenes y Alcohol. Estocolmo, Febrero 2001. EUR/00/5020274/6

Organización Mundial de la Salud (2010). Estrategia mundial para reducir el uso nocivo del alcohol. ISBN. Suiza.

Organización Mundial de la Salud (2011). Primera conferencia de Prevención y promoción de la Salud en la Práctica Clínica en España Prevención de los problemas derivados del alcohol.

Porciel J. (2011). Un Estudio en los Universitarios del Nordeste Argentino Instituto de Criminalistica y Criminologia - UNNE Corrientes - Argentina.

Rojas. M. (2002) Factores de riesgo y protectores identificados en adolescentes consumidores de sustancias psicoactivas. Revisión y análisis del estado actual , en A. Zavaleta, editor: Factores de riesgo y protección en el consumo de drogas en la juventud. Lima: Cedro.

Sánchez Hervas E, Tomas Gradali V, Molina Bou N, del Olmo Guerrea R. Morales Gallus E (2002). Procesos de cambios en conductas adictivas, influencias de variables psicopatologicas y de consumo. Adicciones; 14(3): 337.

Urbina, Dionicio (2008). Desarrollo cerebral y riesgo posterios relacionado al abuso del consumo de alcohol Guadalajara. Mexico. 